\title{
Oral Health Literacy and Related Factors among Pregnant Women Referring to Health Government Institute in Kerman, Iran
}

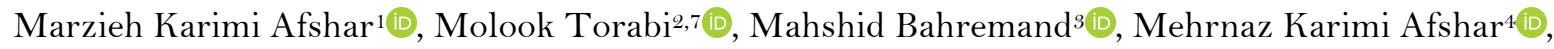 \\ Fatemeh Najmi ${ }^{5}$, Iman Mohammadzadeh ${ }^{6}$ (1)
}

\begin{abstract}
'Department of Orthodontics, School of Dentistry, Kerman University of Medical Sciences, Kerman, Iran. ${ }^{2}$ Kerman Social Determinants on Oral Health Research Center, Kerman University of Medical Sciences, Kerman, Iran. ${ }^{3}$ School of Dentistry, Kerman University of Medical Sciences, Kerman, Iran.

${ }^{4}$ Department of Prosthodontics, School of Dentistry, Tehran University of Medical Sciences, Tehran, Iran.

${ }^{5}$ Department of Community Dentistry, School of Dentistry, Kerman University of Medical Sciences, Kerman, Iran. ${ }^{6}$ Oral and Dental Diseases Research Center, Kerman University of Medical Science, Kerman, Iran.

7Department of Oral Pathology, School of Dentistry, Kerman University of Medical Sciences, Kerman, Iran.
\end{abstract}

Author to whom correspondence should be addressed: Molook Torabi, Department of Oral Pathology, School of Dentistry, Kerman University of Medical Sciences, Shafa Ave, Jomhoori Islami BLVD, Kerman 7618759689, Iran. Phone: +98 9131415238. E-mail: m.torabi.p@,gmail.com.

Academic Editors: Alessandro Leite Cavalcanti and Wilton Wilney Nascimento Padilha

Received: 17 September 2019 / Accepted: 31 October 2019 / Published: 11 November 2019

How to cite this article: Afshar MK, Torabi M, Bahremand M, Afshar MK, Najmi F, Mohammadzadeh I. Oral health literacy and related factors among pregnant women referring to Health Government Institute in Kerman, Iran. Pesqui Bras Odontopediatria Clín Integr. 2020; 20:e5337. https://doi.org/10.1590/pboci.2020.011

\begin{abstract}
Objective: To evaluate oral health literacy among pregnant women in Kerman, Iran. Material and Methods: The present cross-sectional study was conducted on 169 pregnant women referring to government institute. Data were collected by demographic profile (including age, number of children, educational level, dental attendance before pregnancy and economic status), 17-item oral health literacy questionnaire consisting of four domains (including reading comprehension, numeracy, listening, and decision-making skills), self-assessment oral health status, DMFT index and oral health behavior. Data were analyzed by SPSS version 21 software using ANOVA and Chi-square tests at a significance level of 0.05. Results: The mean age of participants was $27.92 \pm 5.25$ years, $38.2 \%$ of them had bachelor's degree, $35.8 \%$ had dental attendance before pregnancy and $29.1 \%$ brushed daily their teeth twice or more. Dentists were the most frequent source of oral health information. Moreover, $60.0 \%$ believed their oral health was to be moderate, and $59.4 \%$ had inadequate oral health literacy. There was also a significant correlation between educational level, monthly income and dental attendance before pregnancy. Conclusion: Our results showed inadequate oral health literacy among pregnant women, highlighting the necessity of dental consultation before pregnancy.
\end{abstract}

Keywords: Oral Health; Health Literacy; Health Behavior; Pregnancy. 


\section{Introduction}

Hormonal changes during pregnancy and non-compliance with oral hygiene increase the incidence of oral diseases in pregnancy [1]. Previous studies have shown the correlation between periodontitis, low birth weight and early delivery [2]. Some studies also suggest that pregnancy elevates the risk of dental caries $[3,4]$. Mothers with poor oral hygiene may be at increased risk of transmission of cariogenic bacteria to their infants $[5,6]$. The history of active caries or decayed tooth cavities in mothers is a predictive factor for early childhood caries [7].

A previous study showed a definite correlation between pregnancy and dental caries, and that pregnant women are at higher risk of caries in the third trimester of pregnancy [8]. The main goal of dental care during pregnancy is to maintain a healthy oral cavity in pregnant women by controlling plaque through brushing, flossing and professional prophylaxis, including scaling, root planing and polishing [9].

Preserving oral health during pregnancy is recognized as one of the most important public health issues in the world. Statements and guidelines have been published, focusing on the emphasis on oral healthcare during pregnancy [10]. Some studies have shown that most pregnant women have received no information on oral health during pregnancy and less than half of them consulted with the dentist [11-14].

"Literacy" represents a set of skills that includes reading, writing, listening, numerical basic calculations and reading comprehension. The concept of "health literacy" was emerged by combining multilateral literacy into health content [15]. In the past decade, the vital role of health literacy has been given special attention in medicine and public health $[16,17]$. The consequences of low health literacy include negative health behaviors, reduced use of preventive services, and poorer use of treatment protocols [18-20].

The most commonly used definition of oral health literacy is "the degree to which individuals have the capacity to obtain, process, and understand basic oral health information and services needed to make appropriate oral health decisions" [21]. This definition addresses the practical literacy of oral health and encompasses the focus on oral health information, guidance to healthcare systems, and active communication with health professionals. It is necessary to achieve health literacy skills to reduce health controversies and improve oral health [22]. The current concept of oral health literacy involves cultural factors and understanding of the knowledge necessary for making healthy oral health decisions [23].

The previous study examined oral health literacy in pregnant women in Brazil and found a positive correlation between the health literacy score with knowledge on the age of consuming sugars in infant feeding. The mean health literacy score was higher in women with more than eight literacy classes. Those who were at a lower social level had lower health literacy [24]. After assessing oral health literacy in American women with the first pregnancy, it was found that their health literacy was low. The health literacy of this group had a significant correlation with their level of knowledge. The authors believe that low oral health knowledge can affect the health of both mother and child [25]. Similar studies have shown that low-educated pregnant women are associated with lower pregnancy and poorer health behaviors [26-30].

Regarding changes in the oral and dental condition during pregnancy, and given that studies underline the importance of oral health literacy, especially during pregnancy, and the lack of research in this context, the current study aimed to investigate the oral health literacy among the pregnant mothers in Kerman

\section{Material and Methods}

Study Design and Sample 
In this cross-sectional study, the target population consisted of 169 pregnant women selected based on sample size formula, referring to health government institute in Kerman, Iran, in January from May 2018.

Exclusion criteria were illiterate women, age before 18 years and reluctant to participate. After selecting the centers and obtaining the necessary permits, the researcher referred to the designated centers three days a week, explained the study objectives to pregnant women, mentioned voluntary participation in the study and the confidentiality of the information, and finally gave the questionnaire to the pregnant women. Participants' selection did through the random method.

\section{Data Collection}

The data collection tool was a questionnaire consisting of three sections. The first part was related to the demographic characteristics, including age, educational level, number of children, and dental attendance before pregnancy, assessment of individual oral health, economic status and assessment of Decayed, Missing, and Filled Teeth (DMFT) indicator.

The second part of the oral health literacy assessment was Oral Health Literacy-Adult Questionnaire (OHL-AQ) in the Persian language. The internal consistency was evaluated using the Cronbach's alpha coefficient of 0.72 and ICC of 0.84 [31]. The questionnaire consists of 17 items, which in four sections examine the reading comprehension, numeracy, listening, and decision-making skills of individuals. The section dealing with reading comprehension had six questions regarding the ability to read and understand the oral health issues, the number of teeth, the age of teeth eruption, the type of toothpaste, the frequency of brushing and dental floss.

The numeracy section evaluated four questions about the numeracy ability of dental prescriptions and instructions for using mouthwashes. In this section, two hypothetical prescriptions were presented to the patient, and questions were asked about the time to take the medication and how to use the mouthwash. The listening section had two questions for evaluating communication skills so that the questioner read the tips for post-extraction care for the person, and then the participant was asked to answer the questions. The decisionmaking section included five questions related to common oral and dental problems that were asked about how to deal with them.

The correct answer in each part was scored with score one and the wrong answer with zero. The total score of the questionnaire was ranged from 0 to 17 , including $0-6$ in the reading comprehension section, $0-4$ in the numeracy section, $0-2$ in the listening section and $0-5$ in the decision-making section. The results were categorized into three groups: 0-9 score as inadequate literacy, 10-11 as moderate, and 12-17 as adequate literacy [33]. The third part of the questionnaire was about oral health behaviors, such as the frequency of brushing and the use of toothpaste, the last visit to the dentist and sources of information on oral health.

\section{Data Analysis}

Data were analyzed by SPSS version 21 software using Chi-square tests at a significance level of 0.05.

Ethical Aspects

This research was approved with the Code of IR.KMU.REC.1396.2151 by the Ethics Committee of Kerman University of Medical Sciences. The participants were assured that their name would not be mentioned in any part of the research and that the participation in the project is completely voluntary and with full consent 


\section{Results}

The mean age of participants was $27.92 \pm 5.25$ years, $33.9 \%$ had a single child, $38.2 \%$ of them had bachelor's degree, $64.2 \%$ patients did not refer to the dentist before making the decision. During pregnancy, $85.5 \%$ patients did not refer to the dentist. The most common source of information was dentists (Table 1).

Table 1. Sample distribution according to demographic variables.

\begin{tabular}{lcc}
\hline \multicolumn{1}{c}{ Variables } & N & \% \\
\hline Number of Children & 87 & 53.7 \\
\hline 1 & 56 & 33.9 \\
2 & 17 & 10.3 \\
3 and 4 & 9 & 5.3 \\
Educational Level & & \\
$\quad$ High School & 10 & 6.1 \\
Diploma & 50 & 30.3 \\
Associate Degree & 17 & 10.3 \\
Bachelor & 63 & 38.2 \\
Higher than a Bachelor's Degree & 22 & 13.3 \\
Attendance to Dentist Before Decision of Pregnancy & & 35.8 \\
Yes & 59 & 64.2 \\
No & 106 & \\
Attendance to Dentist in Pregnancy & & 14.5 \\
Yes & 24 & 85.5 \\
No & 141 & \\
Oral Health Information Source & & 41.8 \\
Dentist & 69 & 26.6 \\
Internet & 45 & 14.8 \\
Family & 25 & 8.9 \\
Radio and TV & 15 & \\
More than One Source & 15 & \\
\hline
\end{tabular}

Regarding tooth brushing, $55.8 \%$ of people toothbrushed once a day, and $4.2 \%$ did not remember the last time of visiting a dentist. In addition, $60 \%$ assessed their oral health as moderate. The correct answer was the best way to remove dental calculus and pigment, 90.3\% responded correctly. The lowest correct answer was related to allergy, responded correctly by $15.8 \%$.

Table 2. Frequency distribution of individuals according to oral health behavior.

\begin{tabular}{lcc}
\multicolumn{1}{c}{ Variables } & $\mathbf{N}$ & \% \\
\hline Tooth Brush Behavior & 7 & 4.2 \\
Once a Week & 18 & 10.9 \\
2-3 Times Weekly & 92 & 55.8 \\
Once a Day & 48 & 29.1 \\
$\quad$ Two Times or More a Day & & \\
Using Tooth Paste & 136 & 82.4 \\
$\quad$ Always & 22 & 13.3 \\
Often & 6 & 3.6 \\
$\quad$ Seldom & 1 & 0.6 \\
$\quad$ Never & & \\
Last Time of Dental Visit & 51 & 30.9
\end{tabular}




\begin{tabular}{lcc} 
6-12 Month Ago & 46 & 27.2 \\
1-2 Years Ago & 40 & 24.2 \\
2-5 Years Ago & 6 & 3.6 \\
Don't Remember & 7 & 4.2 \\
Frequency of Snack & & \\
3 Times or More Daily & 27 & 16.4 \\
2 Times Daily & 39 & 23.6 \\
1 Time Daily & 25 & 15.2 \\
Occasionally & 63 & 38.2 \\
Seldom & 11 & 6.1 \\
\hline
\end{tabular}

The mean DMFT index in the current study was $3.93 \pm 5.64$. The correlation was statistically significant between DMFT and self-assessment oral health status $(\mathrm{p}=0.003)$. People who had a poorly rated oral health had higher DMFT index. The level of health literacy showed 98 (59.4\%) with inadequate literacy and $36(21.8 \%)$ with adequate literacy. Table 3 represents the correlation between educational level, selfassessment of oral health status and dental attendance before pregnancy with oral health literacy. As can be seen, there was no statistically significant correlation between self-assessment oral health status and health literacy level.

Table 3. The relationship between education, monthly income and dental visit before pregnancy and self-assessment of oral health with oral health literacy level.

\begin{tabular}{|c|c|c|c|c|c|c|c|}
\hline \multirow[t]{2}{*}{ Variables } & \multicolumn{2}{|c|}{$\begin{array}{c}\text { Inadequate } \\
\text { Health Literacy }\end{array}$} & \multicolumn{2}{|c|}{$\begin{array}{c}\text { Medium Health } \\
\text { Literacy }\end{array}$} & \multicolumn{2}{|c|}{$\begin{array}{c}\text { Adequate Health } \\
\text { Literacy }\end{array}$} & \multirow[t]{2}{*}{ p-value } \\
\hline & $\mathrm{N}$ & $\%$ & $\mathrm{~N}$ & $\%$ & $\mathrm{~N}$ & $\%$ & \\
\hline \multicolumn{8}{|l|}{ Education } \\
\hline High School & 7 & 4.3 & 2 & 1.2 & 1 & 0.6 & 0.006 \\
\hline Diploma & 40 & 24.7 & 5 & 3.1 & 5 & 3.1 & \\
\hline Associate Degree & 11 & 6.8 & 4 & 2.5 & 2 & 1.2 & \\
\hline Bachelor & 28 & 17.3 & 14 & 8.6 & 21 & 13.0 & \\
\hline Higher than a Bachelor's Degree & 9 & 5.6 & 6 & 3.7 & 7 & 4.3 & \\
\hline \multicolumn{8}{|l|}{ Income* } \\
\hline One Million Tomans & 46 & 30.3 & 4 & 2.6 & 8 & 5.3 & 0.002 \\
\hline 1-2 Million Tomans & 27 & 17.8 & 12 & 7.9 & 12 & 7.9 & \\
\hline 2-3 Million Tomans & 11 & 7.2 & 10 & 6.6 & 10 & 6.6 & \\
\hline More than 3 Million Tomans & 5 & 3.3 & 3 & 2.0 & 2 & 1.3 & \\
\hline \multicolumn{8}{|c|}{ Dental Visit Before Decision to Get Pregnant } \\
\hline No & 76 & 46.1 & 12 & 7.3 & 18 & 10.9 & 0.000 \\
\hline Yes & 22 & 13.3 & 19 & 11.5 & 18 & 10.9 & \\
\hline \multicolumn{8}{|l|}{ Self-Assessment of Oral Health } \\
\hline Very Good & 5 & 3.0 & 1 & 0.6 & 2 & 1.2 & 0.565 \\
\hline Good & 17 & 10.3 & 5 & 3.0 & 11 & 6.7 & \\
\hline Medium & 56 & 33.9 & 22 & 13.3 & 21 & 12.7 & \\
\hline Bad & 10 & 6.1 & 3 & 1.8 & 1 & 0.6 & \\
\hline Very Bad & 7 & 4.2 & $\mathrm{O}$ & 0.0 & 1 & 0.6 & \\
\hline I Don't Know & 3 & 1.8 & 0 & 0.0 & $\mathrm{O}$ & 0.0 & \\
\hline Total & 98 & 59.4 & 31 & 18.8 & 36 & 21.8 & \\
\hline
\end{tabular}

*One Million Tomans $=78 \mathrm{US} \$$.

There was no statistically significant association between age, DMFT and number of children with oral health literacy $(\mathrm{p}>0.05)$.

There was no statistically significant association between the score of oral health literacy questionnaire and the frequency of brushing and toothpaste usage. A statistically significant association was 
found between dental information source and health literacy score and all domains. The individuals who received several sources of information had a better score and had higher oral health literacy $(p=0.004)$.

\section{Discussion}

Poor oral health knowledge can affect the health of both mother and child [25]. Pregnant women with low health literacy have lower knowledge about pregnancy and poorer health behaviors [26-30].

In the present study, $84.9 \%$ of pregnant women were brushing once or twice a day. In Italy, 99.1\% were brushing once a day [24]; this rate was once or twice a day for $83 \%$ of people in another study [32]. As we can see, there are differences with the current study, which can be partly due to differences in the design of studies. It seems that all people, especially vulnerable groups like pregnant women, should be constantly encouraged to take care of their health, including tooth brushing regularly.

In the present study, $27.9 \%$ of pregnant women had dental attendance within six months to one year. Some authors reported that $49.8 \%$ of participants had dental examinations in the past year [24]. Preserving oral health during pregnancy is recognized as one of the most important public health issues in the world. Statements and guidelines have been published, focusing on the emphasis on oral healthcare during pregnancy [10]. It has been shown that people with regular dental attendance perform more prevention measures, including the use of dental floss and toothbrushes. The reason for this is that oral hygiene-induced behaviors become an important part of the training received by the patient during regular dental attendance [33,34].

In the current study, the mean DMFT reported by the participants was $3.93 \pm 5.64$, and a statistically significant association was found between mean DMFT with self-assessment oral health status. Streptococcus mutans and lactobacillus counts have been shown to increase at the end of pregnancy [35]. In addition, dietary changes in early pregnancy, such as sweet snacks, can lead to lower saliva pH (36), hereby increasing DMFT. Microbial flora and maternal oral health function are predictors of neonatal oral health. Therefore, the knowledge and performance of pregnant mothers in oral health is important for pediatric orodental health and can be the key to preventing caries in children.

In the current study, oral health literacy was inadequate in $56.4 \%$ of pregnant women. In a previous study, it was shown that $31.6 \%$ had inadequate literacy and $39.9 \%$ had moderate and $28.5 \%$ lower literacy [37]. However, some authors revealed that 46\% had low health literacy [38]. Among Indian participants, it was found that moderate literacy in $33.1 \%$, lower literacy in $37.6 \%$ and adequate literacy in $29.3 \%$ of subjects [39] and the health literacy was reported to be poor in a group of Iranians [40]. As seen, the percentage of people with inadequate oral health literacy in our study is more than the mentioned studies. The reason for this difference can be in the type of population studied.

The oral health literacy of pediatric nurses had a multidimensional effect on the health of children and infants [41]. It has been shown that poor paternal oral literacy was associated with more caries and less repair of their teeth in their children [42]. Given the role of mothers in pediatric orodental health, more efforts seem to be needed to increase the health literacy of pregnant women. In this study, there was no statistically significant correlation between the self-assessment oral health status and the level of health literacy. This finding is inconsistent with previous reports, which showed a positive correlation between self-assessment oral health status and oral health literacy [43].

In the present study, there was a statistically significant correlation between the level of literacy of individuals and monthly income with oral health literacy. Higher education and higher income status had led to higher health literacy. In a previous study, the level of literacy of the people had a significant correlation 
with the level of oral health literacy [44]. Some authors showed that education and race had a significant correlation with oral health literacy [22,45].

Our findings are in agreement with those reported in the literature [40,46-48]. Higher educational levels seem to lead to more awareness about health and the use of available educational tools, thereby increasing the level of health literacy. In the present study, the age of the subjects did not correlate statistically with the educational level of individuals, which is consistent with the previously described [24]. Some authors have shown that older people had a lower level of literacy [40,49] and, consequently, had worse oral health that was inconsistent with the current study. The reason for this difference can be attributed to the type of study so that the present study was conducted only on pregnant women and the age range was not high. In the present study, the most important source of information on oral health was dentists and then the Internet. The most important source of dental information was the Internet (16\%) and then the dentists (14.8\%) [44]. In the studies in Tehran and Saudi Arabia, the dentists have been listed as the most important source of dental information [40,50]. The dentists are among the key sources in providing information about orodental health and healthy behaviors. Given the growth of Internet access, the collaboration of Orodental Health Custodians with various medical sites seems to be effective in informing the public about orodental health behaviors and raising the oral health literacy of the community.

In this study, the people who came to the dentist before deciding to have a pregnancy had higher health literacy. Considering the development of deciduous teeth and some permanent teeth during embryonic age, dental examinations and counseling are recommended before going to pregnancy.

\section{Conclusion}

A high percentage of pregnant women had inadequate oral health literacy. People with higher educational levels had significantly higher health literacy. It is recommended to encourage women to visit dentistry before pregnancy and to recognize the importance of health during pregnancy.

\section{Authors' Contributions}

$\begin{array}{lll}\text { MKA } & \text { (D) 0000-0003-4485-5476 } & \text { Conceptualization and Validation. } \\ \text { MT } & \text { (D) } 0000-0002-3116-2382 & \text { Methodology, Formal Analysis and Supervision. } \\ \text { MB } & \text { (D) } 0000-0003-3905-9037 & \text { Investigation. } \\ \text { MKA } & \text { (D) } 0000-0002-3440-0198 & \text { Resources and Writing - Original Draft Preparation. } \\ \text { FN } & \text { (D) } 0000-0001-6061-212 \mathrm{X} & \text { Data Curation and Funding Acquisition. } \\ \text { IM } & \text { (D) } 0000-0001-6351-3119 & \text { Writing - Review and Editing and Visualization. } \\ \text { All authors declare that they contributed to critical review of intellectual content and approval of the final version to be } \\ \text { published. }\end{array}$

\section{Financial Support}

None.

\section{Conflict of Interest}

The authors declare no conflicts of interest.

\section{Acknowledgments}

The authors hereby would like to thank and appreciate all the pregnant women who participated in this research and the Deputy of Research and Technology at the Kerman University of Medical Sciences to approve the project. 


\section{References}

[1] Cengiz SB. The pregnant patient: considerations for dental management and drug use. Quintessence Int 2007; 38(3):e133-42.

[2] Xiong X, Buekens P, Fraser WD, Beck J, Offenbacher S. Periodontal disease and adverse pregnancy outcomes: a systematic review. BJOG 2006; 113(2):135-43. https://doi.org/10.1111/j.1471-0528.2005.00827.x

[3] George A, Shamim S, Johnson M, Ajwani S, Bhole S, Blinkhorn A, et al. Periodontal treatment during pregnancy and birth outcomes: A meta-analysis of randomised trials. Int J Evid Based Healthc 2011; 9(2):122-47. https://doi.org/10.1111/j.1744-1609.2011.00210.x

[4] American Academy of Pediatric Dentistry. Clinical Affairs Committee. Infant Oral Health Subcommittee. Guideline on infant oral health care. Pediatr Dent 2012; 34(5):e148-52.

[5] Cucó G, Fernández-Ballart J, Sala J, Viladrich C, Iranzo R, Vila J, et al. Dietary patterns and associated lifestyles in preconception, pregnancy and postpartum. Eur J Clin Nutr 2006; 60(3):364-71. https://doi.org/10.1038/sj.ejcn.1602324

[6] George A, Johnson M, Blinkhorn A, Ajwani S, Bhole S, Yeo AE, et al. The oral health status, practices and knowledge of pregnant women in south-western Sydney. Aust Dent J 2013; 58(1):26-33. https://doi.org/10.1111/adj.12024

[7] Ramos-Gomez FJ, Crystal YO, Wai M, Crall JJ, Featherstone JD. Pediatric dental care: prevention and management protocols based on caries risk assessment. J Calif Dent Assoc 2010; 38(10):746-61.

[8] Kamate WI, Vibhute NA, Baad RK. Estimation of DMFT, salivary streptococcus mutans count, flow rate, pH, and salivary total calcium content in pregnant and non-pregnant women: a prospective study. J Clin Diagn Res 2017; 11(4):ZC147-ZC151. https://doi.org/10.7860/JCDR/2017/24965.9516

[9] Mills LW, Moses DT. Oral health during pregnancy. MCN Am J Matern Child Nurs 2002; 27(5):275-80.

[10] Buerlein J, Peabody H, Santoro K. NIHCM Foundation. Improving Access to Perinatal Oral Health Care: Strategies and Considerations for Health Plans: Issue Brief July 2010 National Institute for Health Care Management. Available from: www.nihcm.org/pdf/NIHCM-OralHealth-Final.pdf. [Accessed on Dec 01, 2014].

[11] Thomas NJ, Middleton PF, Crowther CA. Oral and dental health care practices in pregnant women in Australia: a postnatal survey. BMC PregnancyChildbirth 2008; 8:13. https://doi.org/10.1186/1471-2393-8-13

[12] George A, Johnson M, Blinkhorn A, Ellis S, Bhole S, Ajwani S. Promoting oral health during pregnancy: current evidence and implications for Australian Midwives. J Clin Nurs 2010; 19(23-24):3324-33. https://doi.org/10.1111/j.1365-2702.2010.03426.x

[13] Pitiphat W, Joshipura KJ, Gillman MW, Williams PL, Douglass CW, Rich-Edwards JW. Maternal periodontitis and adverse pregnancy outcomes. Community Dent Oral Epidemiol 2008; 36(1):3-11.

https://doi.org/10.1111/j.1600-0528.2006.00363.x

[14] Oral Health Care During Pregnancy Expert Workgroup. Oral Health Care During Pregnancy: A National Consensus Statement. Washington: National Maternal and Child Oral health Resource Center; 2012.

[15] Nielsen-Bohlman L, Panzer A, Hamlin B, Kindig D. Institute of Medicine. Health literacy: a prescription to end confusion. Committee on Health Literacy, Board on Neuroscience and Behavioral Health. Washington, DC: National Academies Press; 2004.

[16] Protheroe J, Wallace LS, Rowlands G, DeVoe JE. Health literacy: setting an international collaborative research agenda. BMC Fam Pract 2009; 10:51. https://doi.org/10.1186/1471-2296-10-51

[17] Nutbeam D. The evolving concept of health literacy. Soc Sci Med 2008; 67(12):2072-8. https://doi.org/10.1016/j.socscimed.2008.09.050

[18] Dickson-Swift V, Kenny A, Farmer J, Gussy M, Larkins S. Measuring oral health literacy: a scoping review of existing tools. BMC Oral Health 2014; 14:148. https://doi.org/10.1186/1472-6831-14-148

[19] Dewalt DA, Berkman ND, Sheridan S, Lohr KN, Pignone MP. Literacy and health outcomes: a systematic review of the literature. J Gen Intern Med 2004, 19(12):1228-39. https://doi.org/10.1111/j.1525-1497.2004.40153.x

[20] Andrus MR, Roth MT. Health literacy: a review. Pharmacotherapy 2002; 22(3):282-302. https://doi.org/10.1592/phco.22.5.282.33191

[21] Mohammadi TM, Malekmohammadi M, Hajizamani HR, Mahani SA. Oral health literacy and its determinants among adults in Southeast Iran. Eur J Dent 2018; 12(3):439-42. https://doi.org/10.4103/ejd.ejd_429_17

[22] Horowitz AM, Kleinman DV. Oral health literacy: a pathway to reducing oral health disparities in Maryland. J Public Health Dent 2012; 72(Suppl 1):S26-30. https://doi.org/10.1111/j.1752-7325.2012.00316.x

[23] Burgette JM, Lee JY, Baker AD, Vann WF Jr. Is dental utilization associated with oral health literacy? J Dent Res 2016; 95(2):160-6. https://doi.org/10.1177/0022034515617457

[24] Vilella KD, Alves SG, de Souza JF, Fraiz FC, Assunção LR. The association of oral health literacy and oral health knowledge with social determinants in pregnant Brazilian women. J Community Health 2016; 41(5):1027-32. https://doi.org/10.1007/s10900-016-0186-6

[25] Hom JM, Lee JY, Divaris K, Baker AD, Vann WF Jr. Oral health literacy and knowledge among patients who are pregnant for the first time. J Am Dent Assoc 2012; 143(9):972-80. https://doi.org/10.142 19/jada.archive.2012.0322 
[26] Arnold CL, Davis TC, Berkel HJ, Jackson RH, Nandy I, London S. Smoking status, reading level, and knowledge of tobacco effects among low-income pregnant women. Prev Med 2001; 32(4):313-20. https://doi.org/10.1006/pmed.2000.0815

[27] Cho RN, Plunkett BA, Wolf MS, Simon CE, Grobman WA. Health literacy and patient understanding of screening tests for aneuploidy and neural tube defects. Prenat Diagn 2007; 27(5):463-7. https://doi.org/10.1002/pd.1712

[28] Gazmararian JA, Parker RM, Baker DW. Reading skills and family planning knowledge and practices in a lowincome managed-care population. Obstet Gynecol 1999; 93(2):239-44. https://doi.org/10.1016/s0029-7844(98)00431-1

[29] Endres LK, Sharp LK, Haney E, Dooley SL. Health literacy and pregnancy preparedness in pregestational diabetes. Diabetes Care 2004; 27(2):331-4. https://doi.org/10.2337/diacare.27.2.331

[30] Shieh C, Mays R, McDaniel A, Yu J. Health literacy and its association with the use of information sources and with barriers to information seeking in clinic-based pregnant women. Health Care Women Int 2009; 30(11):971-88. https://doi.org/10.1080/07399330903052152

[31] Naghibi Sistani MM, Montazeri A, Yazdani R, Murtomaa H. New oral health literacy instrument for public health: development and pilot testing. J Investig Clin Dent 2014; 5(4):313-21. https://doi.org/10.1111/jicd.12042

[32] Boggess KA, Urlaub DM, Massey KE, Moos MK, Matheson MB, Lorenz C. Oral hygiene practices and dental service utilization among pregnant women. J Am Dent Assoc 2010; 141(5):553-61. https://doi.org/10.14219/jada.archive.2010.0228

[33] Astrøm AN, Ekback G, Ordell S, Nasir E. Long-term routine dental attendance:influence on tooth loss and oral health-related quality of life in Swedish older adults. Community Dent Oral Epidemiol 2014; 42(5):460-9. https://doi.org/10.1111/cdoe.12105

[34] Rahman B, Al Kawas S. The relationship between dental health behavior, oral hygiene and gingival status of dental students in the United Arab Emirates. Eur J Dent 2013; 7(1):22-7.

[35] Pirie M, Cooke I, Linden G, Irwin C. Dental manifestations of pregnancy. Obstet Gynaecol 2007; 9(1):21-6. https://doi.org/10.1576/toag.9.1.021.27292

[36] Jain K, Kaur H. Prevalence of oral lesions and measurement of salivary pH in the different trimesters of pregnancy. Singapore Med J 2015; 56(1):53-7. https://doi.org/10.11622/smedj.2015010

[37] Ueno M, Takeuchi S, Oshiro A, Kawaguchi Y. Relationship between oral health literacy and oral health behaviors and clinical status in Japanese adults. J Dent Sci 2013; 8(2):170-6. https://doi.org/10.1016/j.jds.2012.09.012

[38] Jones K, Brennan DS, Parker EJ, Mills H, Jamieson L. Does self-efficacy mediate the effect of oral health literacy on self-rated oral health in an Indigenous population? J Public Health Dent 2016; 76(4):350-5. https://doi.org/10.1111/jphd.12162

[39] Sandhu KS, Gupta N, Bansal M, Arora V, Gupta P, Thakar S. Health literacy and oral health literacy: are they associated? A cross sectional survey among undergraduate students of tricity (Chandigarh, Mohali, Panchkula), India. Przegl Epidemiol 2017; 71(1):111-8.

[40] Sistani MM, Yazdani R, Virtanen J, Pakdaman A, Murtomaa H. Oral health literacy and information sources among adults in Tehran, Iran. Community Dent Health 2013; 30(3):178-82.

[41] Vann WF, Lee JY, Baker D, Divaris K. Oral health literacy among female caregivers: impact on oral health outcomes in early childhood. J Dent Res 2010; 89(12):1395-1400. https://doi.org/10.1177/0022034510379601

[42] Khodadadi E, Niknahad A, Naghibi Sistani MM, Motallebnejad M. Parents' oral health literacy and its impact on their children's dental health status. Electron Physician 2016; 8(12):342 1-5. https://doi.org/10.19082/342 1

[43] Guo Y, Logan HL, Dodd VJ, Muller KE, Marks JG, Riley JL 3rd. Health literacy: a pathway to better oral health. Am J Public Health 2014; 104(7):e85-91. https://doi.org/10.2105/AJPH.2014.301930

[44] Afshar MK, Torabi M, Khosravi M. Association between oral health literacy and self-reported oral health behavior in adults( Kerman-Iran). J Fundam Appl Sci 2018; 10(6S):2813-25.

[45] Martin LT, Ruder T, Escarce JJ, Ghosh-Dastidar B, Sherman D, Elliott M, et al. Developing predictive models of health literacy. J Gen Intern Med 2009; 24(11):1211-6. https://doi.org/10.1007/s1 1606-009-1 105-7

[46] Roncalli AG, Sheiham A, Tsakos G, Watt RG. Socially unequal improvements in dental caries levels in Brazilian adolescents between 2003 and 2010. Community Dent Oral Epidemiol 2015; 43(4):317-24. https://doi.org/10.1111/cdoe.12156

[47] Jones M, Lee JY, Rozier RG. Oral health literacy among adult patients seeking dental care. J Am Dent Assoc 2007; 138(9):1 199-208. https://doi.org/10.14219/jada.archive.2007.0344

[48] Atchison KA, Gironda MW, Messadi D, Der-Martirosian C. Screening for oral health literacy in an urban dental clinic. J Public Health Dent 2010; 70(4):269-75. https://doi.org/10.1111/j.1752-7325.2010.00181.x

[49] Javadzade SH, Sharifirad G, Radjati F, Mostafavi F, Reisi M, Hasanzade A. Relationship between health literacy, health status, and healthy behaviors among older adults in Isfahan, Iran. J Educ Health Promot 2012; 1:31. https://doi.org/10.4103/2277-9531.100160

[50] Wyne AH, Chohan AN, Al-Dosari KH, Al-Dokheil MA. Oral health knowledge and sources of information among male Saudi school children. Odontostomatol Trop 2004; 27(106):22-6. 